\title{
Coelomic Liposarcoma In An African Pygmy Goose (Nettapus Auritus)
}

\author{
Jason D Struthers ${ }^{1^{*}}$ and Geoffrey W Pye ${ }^{2}$ \\ ${ }^{1}$ From the Animal Health Institute, Department of Pathology and Population Medicine, 5725 W. Utopia Rd., Midwestern University, \\ Glendale, Arizona 85308, USA.
}

${ }_{2}^{2}$ Animals, Science, and Environment, Disney's Animal Kingdom, 1200 N Savannah Circ, Bay Lake, Florida 32830, USA.

Received: 25 May, 2018; Accepted: 11 June, 2018; Published: 12 June, 2018

*Corresponding author: : Jason D. Struthers,From the Animal Health Institute, Department of Pathology and Population Medicine, 5725 W. Utopia Rd., Midwestern University, Glendale, Arizona 85308, USA. E-mail: jstrut@midwestern.edu

\begin{abstract}
A morbid African pygmy goose (Nettapus auritus) developed openmouth breathing and died during physical exam. Necropsy revealed bacterial salpingitis and a coelomic liposarcoma. Death resulted from a combination of poor body condition, infection, stress of handling, and compromised respiratory and cardiovascular function related to the coelomic liposarcoma.
\end{abstract}

Keywords: coelom; duck; liposarcoma; Nettapus auritus; oil red 0 ; pygmy goose

\section{Introduction}

A zoo-born six-year old female African pygmy goose (Nettapus auritus) was found recumbent and lethargic in her enclosure. The bird was depressed, underweight (2/9 BCS; $202.2 \mathrm{~g}$ ), dehydrated, and had pale mucous membranes. Prior to diagnostics, $15 \mathrm{ml}$ of combined Lactated Ringers Solution with $5 \%$ dextrose and $30 \mathrm{mg} / \mathrm{kg}$ enrofloxacin was administered subcutaneously in the left inguinal region. During the therapy, the patient acutely developed open-mouth breathing and died shortly thereafter despite resuscitation attempts. The patient was submitted for necropsy. The patient's previous medical history was unremarkable. The most recent wellness exam occurred fourteen months previously-a physical exam, complete blood count, avian biochemistry panel, and whole body radiographs were considered normal. At that time, the bird weighed $281 \mathrm{~g}$, had a normal body condition score (5/9), and was deemed healthy. A year previously, a fecal float and a fecal direct microscopic exam were unremarkable.

At necropsy, this bird had marked atrophy of the pectoral muscles with no detectable adipose stores. Occupying approximately $70 \%$ of the left ventrocaudal coelom was a $6.5 \times 4 \times 3$ $\mathrm{cm}$, well-demarcated, soft, greasy, pale tan mass with a ventral superficial $2 \times 2 \mathrm{~cm}$ focus of hemorrhage (Figure 1). The spaceoccupying mass caused: a cranial displacement of the heart, lungs, and liver; a dorsal displacement of the kidneys; a lateral displacement of the gastrointestinal tract; a ventral displacement of the caudal aspect of the sternum; and a caudoventral displacement of the cloaca and the oviduct. The mass closely abutted many tissues, including the ventriculus, kidney, oviduct, and, most closely, the cloaca. The mass was dissected and isolated from the surrounding viscera. On section, the mass was greasy, soft, and mottled tan to light red with occasional grey firm areas (necrosis). The oviduct's serosa was diffusely grey to light brown and was markedly distended by soft to granular, grey to brown viscid material. A swab of the lumen was submitted for aerobic bacterial culture.

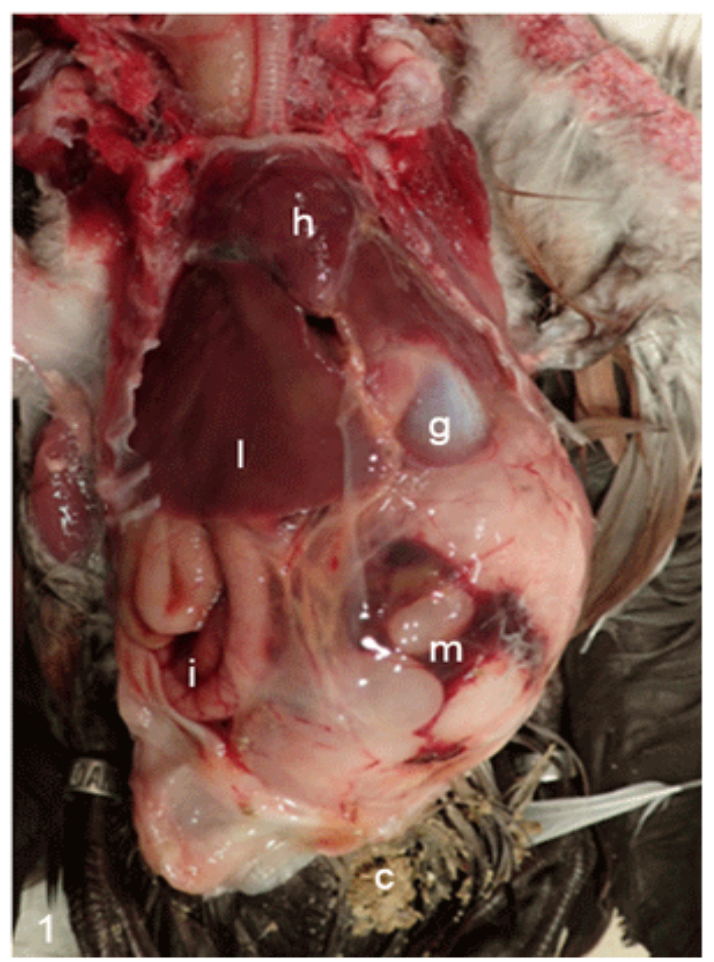

Figure 1: Liver (l), heart (h), intestine (i), coelomic mass (m), cloaca (c), gizzard (g); six-year-old female African pygmy goose (Nettapus auritus). The mass occupies at least $70 \%$ of the coelomic cavity and displaces and abuts the surrounding viscera. The ventral aspect of the mass has regional superficial hemorrhage. The pericloacal feathers are soiled and matted with dried feces and urates 
Histologically, the mass was composed of interlacing streams of loosely organized spindle to polygonal cells separated by clear space and supported by sparse fibrovascular tissue that reached tumor margins or abutted and infiltrated adjacent air sacs, fascicles of skeletal muscle, and bands of dense fibrous connective tissue. Cells had moderate amounts of eosinophilic granular cytoplasm with occasional single large or multiple small, discrete, clear vacuoles that variably marginalized nuclei (Figure 2). Nuclei were round to oval and occasionally cleaved or

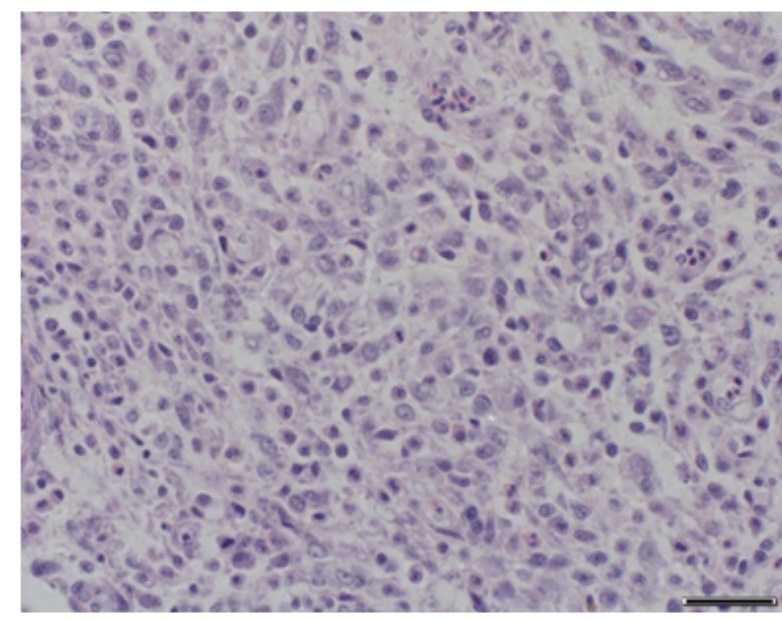

Figure 2: Six-year-old female African pygmy goose (Nettapus auritus), coelomic mass. Spindle to polygonal neoplastic cells with typically single, well-defined, clear intracytoplasmic vacuoles that occasionally marginalize the nucleus. Hematoxylin and eosin stain. Bar $=20 \mu \mathrm{m}$.

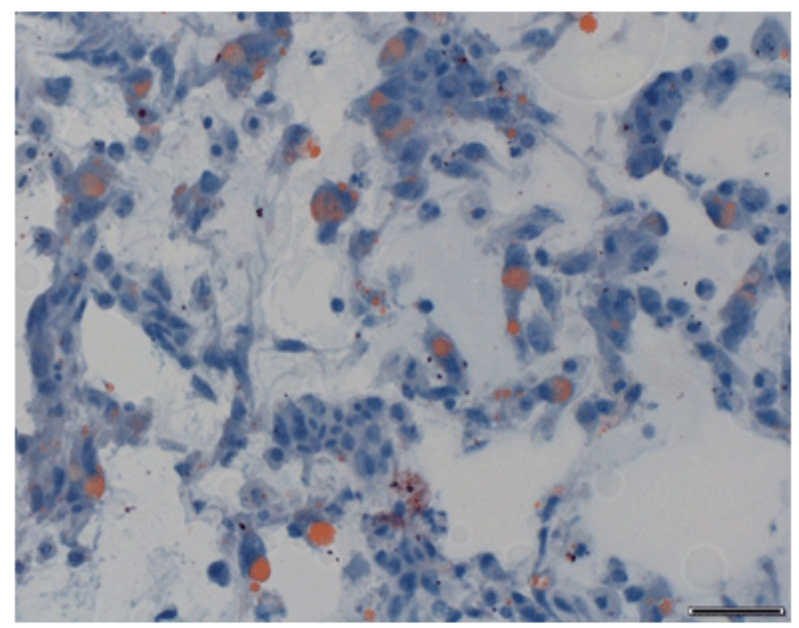

Figure 3: Six-year-old female African pygmy goose (Nettapus auritus), coelomic mass. Neoplastic cell intracytoplasmic vacuoles stain positive for lipid. Oil red 0 histochemical stain. $\operatorname{Bar}=20 \mu \mathrm{m}$.

distorted with up to four dark nucleoli. Nuclear and cytoplasmic pleomorphism was moderate, with occasional binucleation and giant cells with karyomegaly. Mitoses were four in ten $400 \mathrm{x}$ fields. In sections, up to $40 \%$ of the mass was effaced by necrosis, fibrin, and hemorrhage. In some sections, the mass displaced peripheral aggregates of atrophied fat and had loose infiltrates of heterophils, macrophages, lymphocytes, and plasma cells. Other significant microscopic findings included fibrinonecrotic, heterophilic, and histiocytic salpingitis with luminal hemorrhage, debris, and yolk; bilateral compressive pulmonary atelectasis; non-suppurative portal hepatitis with intraductal presumptive trematodes; ventricular koilin degeneration with intralesional cocci; diffuse atrophy of adipose tissue; and pancreatic fibrosis with zymogen granule depletion.

An oil red 0 histochemical stain was performed on formalinfixed sections of tumor. Neoplastic cells had occasional positive oil red 0 staining of well-defined intracytoplasmic vacuoles (Figure 3). Aerobic bacterial culture of the oviduct lumen yielded heavy growth of mixed bacteria, including alpha-hemolytic Streptococcus sp., Citrobacter freudii, Micrococcus sp., and Enterococcus sp.

\section{Discussion}

The most important necropsy finding was a locally extensive and space-occupying coelomic mass. The primary differential diagnosis was a neoplasm. The color, texture, and greasy appearance of the mass were suggestive of a tumor of adipose tissue. Moreover, the lack of infiltration of the surrounding viscera suggested that the primary origin was coelomic adipose tissue. Histopathology was consistent with a sarcoma and in conjunction with positive oil red 0 staining of intracytoplasmic vacuoles, a diagnosis of a poorly-differentiated liposarcoma was made, one with features of both the myxoid variant and the pleomorphic form $[1,2]$. The histologic appearance was similar to liposarcoma reported in the musculature of a goose and a white leghorn chicken $[3,4]$.

Multifocally within the mass, the stroma and neoplastic cells were separated and displaced by random, variably-sized, and poorly-demarcated clefts of clear space. This appearance was believed to be iatrogenic, resulting from the inadvertent injection of fluid into the mass. Due to the size of the coelomic mass and its opposition to the coelomic wall of the left inguinal region, it is believed that the antemortem administered fluid was injected into the coelom (mass) rather than in the subcutis. This theory was supported by the microscopic appearance of the mass, as well as the lack of edema in the subcutis of the area intended for the fluid.

Lethargy, depression, weight loss (approximately $80 \mathrm{~g}$, 28\% of body weight, in 1.2 years) and oviduct pathology can be attributed to the coelomic liposarcoma. The progressive growth of the space-occupying mass would have collapsed abdominal air sacs and put pressure on adjacent viscera, plausibly impacting digestive, cardiovascular, and respiratory function. Aggravated by the stress of handling, it is hypothesized that this bird succumbed to respiratory failure due to decreased air sac and lung capacity as a result of compression by the neoplasm. The pericloacal soiling and the oviduct pathology may reflect the mass' regional pressure on the cloaca, thereby interfering with defecation and predisposing to an ascending mixed bacterial salpingitis. 
Neoplasms are well described in birds and have been diagnosed in at least 22 avian orders. They are documented in all systems and occur in most anatomical locations $[1,5,6]$. Reports have suggested an increased frequency of tumors of the integument and multicentric lymphoma [1,7]. Lipomas are among the most common integumentary tumors, and may also occur in the coelom [1]. Comparatively, liposarcomas are infrequent, and in avian patients they have been described in the subcutis, especially of the sternum and in the area of uropygial gland, and rarely in the coelom [1]. Intracoelomic liposarcomas have been documented in two pigeons, a cockatiel, a chukar partridge, and now in an African pygmy goose [7,8]. Although rare, coelomic liposarcomas may cause morbidity and may predispose to mortality, and thus should be part of the differential diagnosis of a coelomic mass in an avian patient.

\section{Acknowledgments}

We thank Dr. Lisa Farina for commentary and critical review of this manuscript. Also, we would like to acknowledge Disney's Animal Kingdom medical and husbandry teams that every day provides exemplary care for the animals.

\section{References}

1. Harrison G J,Harrison L R, RitchieBW. Avian medicine: principles and applications. Lake Worth, FLa: Wingers Publishing.1994;640-672.

2. Doria-Torra G, Martinez J, Domingo M, Vidana B, Isidoro-Ayza M, Casanova MI,et al. Liposarcoma in animals: literature review and case report in a domestic pig (Sus scrofa). J Vet Diagn Invest.2015;27(2):196-202. doi: 10.1177/1040638714567190

3. Doster AR, Johnson JL, Duhamel GE, Bargar TW, Nason G. Liposarcoma in a Canada goose (Branta canadensis). Avian Dis 1987;31(4):918-920.

4. Mohiddin S M, Ramakrishna K. Liposarcoma in a fowl. Avian Dis. 1972;16(3):680-684.

5. Drury R R. Tumors of pet birds. Vet Clin Exot Anim 2004;7 (3):537560. doi : 10.1016/j.cvex.2004.04.008

6. Lightfoot TL, Garner MM. Overview of tumors: section I and II. In: Harrison G, Lightfoot T, eds. Clinical Avian Medicine. Palm Beach, FL: Spix Publishing, Inc. 2006:559-572.

7. Reece R L . Observations on naturally occurring neoplasms in birds in the state of Victoria, Australia. Avian Pathol 1990;21:3-32.

8. Shimonohara N, Holland C H, Lin T, Wigle W L. Naturally occurring neoplasms in pigeons in a research colony: a retrospective study. Avian Dis.2013;57(1):133-139. 